\title{
Avaliação pericial do ruído em oficina de locomotivas situada na cidade de São Paulo
}

\author{
Expert evaluation of noise at a train manufacturer \\ in the city of Sao Paulo
}

\author{
Sabrina Yumico Akatsu', Eduardo Costa Sá2
}

\begin{abstract}
Akatsu SY, Sá EC. Avaliação pericial do ruído em oficina de locomotivas situada na cidade de São Paulo. Saúde, Ética \& Justiça. 2013;18(1):124-7.

RESUMO: INTRODUÇÃO: A Perda Auditiva Induzida pelo Ruído (PAIR) é uma patologia insidiosa, cumulativa, que cresce ao longo dos anos de exposição ao ruído associado ao ambiente de trabalho e destaca-se como uma das patologias mais prevalentes nas indústrias brasileiras. Relaciona-se a fatores como idade, tempo de exposição, nível de ruído, contato com agentes químicos, exposição anterior ao ruído e cirurgias ou patologias otológicas prévias. OBJETIVO: Propor uma metodologia de avaliação pericial do ruído em uma oficina de locomotivas em São Paulo a partir de uma revisão bibliográfica. MATERIAL E MÉTODOS: revisão bibliográfica sobre o tema Perda auditiva induzida pelo ruído, mediante estudos de artigos científicos encontrados por pesquisa na Base de Dados da Bireme e da Scielo referentes ao período de 1988 a 2010 e análise de um levantamento referente ao ruído do ano de 2010, realizado na oficina de locomotivas de São Paulo. RESULTADOS E DISCUSSÃO: após avaliação das bibliografias e análise do levantamento do ruído, foi proposto um estudo sobre o ruído na oficina. CONLUSÃO: a metodologia proposta para a avaliação pericial do ruído foi a realização de um levantamento de ruído que mencionou e considerou o tipo de levantamento, a escolha de quais funcionários seriam avaliados, dentre outros critérios.
\end{abstract}

DESCRITORES: Ruído ocupacional; Perda auditiva provocada por ruído; Exposição ocupacional.

1. Médica, graduada pela Faculdade de Ciências Médicas de Santos, pós- graduada em Medicina do Trabalho pela Faculdade de Ciências Médicas da Santa Casa de São Paulo e pós-graduada em Medicina Legal/ Perícia Médica pela Faculdade de Ciências Médicas da Santa Casa de São Paulo. Endereço: Rua Dona Veridiana, 484, apto 42 C. E-mail: satikoxli@yahoo.com.br

2. Mestre - Professor e orientador do Curso de Medicina Legal/Perícia Médica da Santa Casa de Misericórdia de São Paulo, Médico do Trabalho e Oftalmologista. E-mail: eduardocs6@gmail.com

Endereço para correspondência: Eduardo Costa Sá. Rua Teodoro Sampaio, 115 - São Paulo, SP. E-mail: eduardocs6@gmail.com 


\section{INTRODUÇÃO}

$\mathbf{O}$ agente rú́do, de um modo geral, constitui-se em um dos maiores riscos potenciais para a saúde dos trabalhadores, tanto nas instalações industriais como em outras atividades laborais. Do ponto de vista da física, o ruído é, por definição, a variação de pressão sonora sob a forma de ondas mecânicas, que apresentam oscilações dos sistemas de materiais elásticos ${ }^{1}$.

A Perda Auditiva Induzida por Ruído (PAIR) é uma patologia reconhecida pela Legislação Brasileira e, para a avaliação da insalubridade por ruído em locais de trabalho, a Norma Regulamentadora $15^{8}$ estabelece os limites de exposição ao ruído para trabalhadores, visando protegê-los de danos auditivos ${ }^{3}$.

Segundo Guida et $\mathrm{al}^{2}$, o ruído, isoladamente, apresenta perigo à saúde quando o nível sonoro é superior a $85 \mathrm{~dB}$, dependendo da duração e exposição sistemática a ele. Por esta razão, a partir desta intensidade sonora, faz-se audiometria, periodicamente em indústrias.

Sabe-se que a Perda Auditiva Induzida por Ruído (PAIR) sucede-se pela exposição sistêmica e prolongada ao ruído intenso; é uma doença crônica e irreversível, uma vez que acomete as células ciliadas do órgão de Corti². Essa afecção acomete, inicialmente, as regiões das frequências de 6,4 ou $3 \mathrm{kHz}$, podendo, com a progressão da perda, atingir regiões de $8,2,1 \mathrm{kHz}, 500$ e $250 \mathrm{~Hz}$.

Segundo Brevigliero et al. ${ }^{1}$, existem dois tipos de levantamento de ruído: o ambiental, que é feito ponto a ponto, com medições instantâneas para conhecimento do perfil do ruído na área (rastreamento) e o pessoal, que é feito através de dosimetria de ruído para avaliação de funções, sendo geralmente feito por meio de audiodosímetros, fixados no trabalhador.

O levantamento do ruído e os dados encontrados em estudos tornam-se importantes meios de comparação com os limites de tolerância estabelecidos pela legislação brasileira, como, por exemplo, os Anexos 1 e 2 da NR15, objetivando prevenir o dano auditivo.

\section{OBJETIVOS}

\section{Principal}

Propor uma metodologia de avaliação pericial do ruído em uma oficina de locomotivas em São Paulo a partir de uma revisão bibliográfica.

\section{Específico}

Avaliar a medição do ruído na jornada de trabalho dos funcionários de uma oficina de locomotivas, durante o ano de 2010, situada na cidade de São Paulo, sob o ponto de vista pericial.

Identificar as principais categorias de trabalhadores que estão expostos ao ruído e os níveis de exposição mais elevados numa empresa de oficina de locomotivas de São Paulo, Brasil.

\section{MATERIAL E MÉTODO}

Este é um estudo transversal realizado em trabalhadores de uma oficina de locomotivas de uma concessionária que controla, opera e monitora a Malha Sudeste da Rede Ferroviária Federal. Após aprovação pelo responsável pela engenharia e segurança do trabalho da empresa em estudo, foi feita uma análise dos dados do levantamento do ruído. Foram avaliados funcionários do setor oficina, que exercem atividade laboral em dois tipos de locomotivas existentes na empresa, e os funcionários do setor administrativo, que estão situados em prédios próximos às oficinas. $\mathrm{Na}$ avaliação, foi selecionado, aleatoriamente, um funcionário de cada função (total de 12 funcionários), com diferentes idades e tempo de serviço e de ambos os sexos. Não foi excluída da análise, nenhuma função. As medições dos níveis de ruído foram realizadas no mês de Janeiro de 2010. Os equipamentos utilizados foram um dosímetro QUEST, modelo Edge eg-4, e um calibrador acústico QUEST QC-10/QC-20. O tempo de leitura do dosímetro variou de acordo com cada função e foi fixado no próprio trabalhador (levantamento do tipo pessoal).

Para o desenvolvimento de uma metodologia que propõe a avaliação pericial do ruído em uma oficina de locomotivas em São Paulo, o presente estudo foi realizado a partir de uma revisão bibliográfica sobre o tema ruído em local de trabalho, no ano de 2011, com seis referências encontradas ${ }^{2-7}$. Foram utilizados para a pesquisa: livros texto sobre Segurança e Medicina do Trabalho e Higiene Ocupacional ${ }^{1,9}$, além de dados de sites do Ministério da Previdência Social ${ }^{11}$, Ministério da Saúde ${ }^{13}$, Ministério do Trabalho ${ }^{10}$ e da Associação Nacional de Medicina do Trabalho (ANAMT) ${ }^{12}$. Também foram utilizados os dados do levantamento referentes ao ruído da oficina de locomotivas de São Paulo, no ano de 2010, que avaliou a medição do ruído na jornada de trabalho dos funcionários da empresa, sob o ponto de vista pericial.

\section{RESULTADO E DISCUSSÃO}

$\mathrm{Na}$ revisão bibliográfica, foram encontrados os seguintes estudos:

Fernandes ${ }^{3}$ : realizou um estudo ambiental sobre o ruído em uma indústria injetora de plásticos e constatou que o galpão não se configurava como um ambiente insalubre.

Macedo et al. ${ }^{4}$ : mensuraram os níveis de pressão sonora de três Unidades de Terapia Intensiva de um 
hospital em Jundiaí e verificaram que nenhuma das três UTI apresentaram níveis maiores que $85 \mathrm{~dB}(\mathrm{~A})$.

Cordeiro et al. $^{5}$ : verificaram que a exposição ocupacional ao ruído é fator de risco relevante para acidentes do trabalho.

Guida et al. ${ }^{2}$ : verificaram que os achados audiológicos em trabalhadores expostos ao ruído ocupacional e a praguicidas eram piores que os achados audiológicos de trabalhadores expostos apenas ao ruído.

De Faria e Suzuki ${ }^{6}$ : verificaram que a atenuação sonora do equipamento de proteção individual (EPI) tipo plugue, utilizado por colaboradores expostos ao ruído, possui nível de atenuação sonora eficaz e compatível com a fornecida pelo fabricante.
Harger et al.7: concluíram que a prevalência de dano auditivo em marmorarias do Distrito Federal foi de $48 \%$ de uma amostra de 152 trabalhadores.

Após avaliação das bibliografias supracitadas, foi proposto um levantamento do tipo pessoal, no qual o dosímetro foi fixado ao corpo (ombro) do trabalhador e foi feita uma análise em todos os postos de trabalho.

Para a avaliação, foi selecionado aleatoriamente um funcionário de cada função; funcionários com diferentes idades e tempo de serviço e de ambos os sexos. As medições dos níveis de pressão sonora foram realizadas no mês de janeiro de 2010. O tempo de leitura dos dosímetros variou de acordo com a função de cada trabalhador (Tabela 1).

Tabela 1. Distribuição das funções dos trabalhadores com os respectivos tempo de medição (min), tempo de pausa (min), NEN $\mathrm{dB}(\mathrm{A})^{*}$ e jornada de trabalho $(\mathrm{min})$

\begin{tabular}{|l|r|r|r|r|}
\hline FUNÇÃO & TEMPO DE MEDIÇÃO (min) & PAUSA(min) & NEN dB(A)* JORNADA( min) \\
\hline mantenedor líder diesel (oficina) & 444 & 74 & $\mathbf{8 9 , 3}$ & 480 \\
\hline téc de manutenção diesel (oficina) & 443 & 76 & 80,6 & 480 \\
\hline mant mecânico diesel (oficina) & 472 & 45 & $\mathbf{9 0 , 7}$ & 480 \\
\hline analista ferroviário (adm) & 405 & 75 & 67,1 & 480 \\
\hline mant eletricista diesel ( oficina) & 458 & 60 & 93,4 & 480 \\
\hline técnico químico (oficina) & 447 & 78 & 84,8 & 480 \\
\hline téc de seg do trabalho(adm + oficina & 392 & 62 & 78,7 & 480 \\
\hline mant líder hitachi (oficina) & 373 & 64 & 84,7 & 480 \\
\hline mant mecânico hitachi (oficina) & 388 & 69 & 98,8 & 480 \\
\hline mant eletricista hitachi (oficina) & 426 & 62 & 93,0 & 480 \\
\hline téc de manutenção hitachi (oficina) & 439 & 79 & 93,0 & 480 \\
\hline comprador (adm) & 405 & 75 & 70,6 & 480 \\
\hline
\end{tabular}

$\mathrm{NEN} \mathrm{dB(A)*} \mathrm{=} \mathrm{nível} \mathrm{de} \mathrm{exposição} \mathrm{normalizado} \mathrm{(nível} \mathrm{de} \mathrm{exposição} \mathrm{convertido} \mathrm{para} \mathrm{a} \mathrm{jornada} \mathrm{padrão} \mathrm{de} 8$ horas).

Para todos os trabalhadores, o tipo de exposição foi habitual e permanente, e as fontes geradoras de ruído foram consideradas de acordo com cada função. A utilização de protetores auditivos adequados é de suma importância, principalmente devido a sua eficácia ao atenuar os ruídos externos, como comprovado no estudo de De Faria e Suzuki ${ }^{6}$. Em nossa análise, todos os trabalhadores referiram utilizar o protetor auricular de inserção durante a jornada de trabalho.

Observamos, no estudo, que nos cargos de mantenedor mecânico, mantenedor eletricista, mantenedor líder e técnico de manutenção encontram-se os funcionários que mais preocupam a área da Saúde e Segurança do Trabalho da empresa, pois são os trabalhadores que ficam durante toda a sua jornada expostos ao ruído acima do permitido pela NR15 (85 dB (A) em 8h); por isso, justificase a necessidade de um maior investimento em programas voltados para o controle de ruídos.

\section{CONCLUSÃO}

A metodologia proposta para a avaliação pericial do ruído na oficina de locomotivas de São Paulo foi a realização de um estudo do ruído que mencionou e considerou o tipo de levantamento, a escolha de quais funcionários seriam avaliados, relatando a ausência de critérios de exclusão a partir de características como: idade, sexo ou função; o período de medição do ruído e o período de pausa; o tipo de jornada de trabalho; o modelo do EPI (Equipamento de Proteção Individual) utilizado pelos trabalhadores e o local onde os trabalhadores realizam a maior parte das atividades laborais.

A avaliação da medição de ruído na jornada de trabalho dos funcionários da oficina de locomotivas, sob o ponto de vista pericial, nos permitiu observar que seis dos nove trabalhadores que permanecem durante toda a sua jornada de trabalho exclusivamente nas oficinas $(66,6 \%)$, estão expostos a níveis de pressão sonora acima do limite de tolerância estabelecido pelo Anexo 1 da NR15.

As principais categorias de trabalhadores que estão expostos aos níveis de pressão sonora mais elevados na oficina de locomotivas na cidade de São Paulo são os de mantenedor mecânico - Hitachi (98,8 dB(A)), mantenedor mecânico - Diesel (90,7 dB(A)), mantenedor eletricista - Diesel $(93,4 \mathrm{~dB}(\mathrm{~A}))$, mantenedor eletricista - Hitachi (93 dB(A)), mantenedor líder - Diesel (89,3 $\mathrm{dB}(\mathrm{A}))$, e técnico de manutenção - Hitachi (93 dB(A)). 
AGRADECIMENTOS: Ao Prof. Dr. Daniel Romero Munõz, professor Titular da Disciplina de Medicina Legal/Perícias Médicas da Faculdade de Ciências Médicas da Santa de Misericórdia de São Paulo e responsável pelo Curso de Pós-Graduação em Medicina Legal/Perícias médicas, minha admiração e sincera gratidão pela oportunidade de freqüentar este curso e por todo conhecimento adquirido ao longo do mesmo. À Faculdade de Ciências Médicas da Santa Casa de São Paulo e à Irmandade da Santa Casa de Misericórdia de São Paulo.

Akatsu SY, Sá EC. Expert evaluation of noise at a train manufacturer in the city of Sao Paulo. Saúde, Ética \& Justiça. 2013;18(1):124-7.

\begin{abstract}
INTRODUCTION: Noise-Induced Hearing Loss (NIHL) is a cumulative pathological disease that increases over the years with exposure to noise associated with the work atmosphere and is targeted as one of the most prevalent diseases in Brazilian industries. It is associated to the increasing of age, exposure time to loud noise, noise level, contact with chemical agents, previous exposure to noise and previous surgeries or previous otologic pathologies. OBJECTIVE: To Propose a methodology for expert evaluation of noise at a train manufacturer manufactory, in São Paulo, from a literature review. MATERIAL AND METHODS: a literature review about noise induced hearing loss, searched on the Bireme and SciELO databases during the period of 1988 to 2010 and analysis of a study that measured the workers' exposure to noise at a train manufacturer. RESULTS AND DISCUSSION: After study of noise by way literature review and analysis, a study methodology was proposed. CONCLUSION: The methodology proposed for a methodology for expert evaluation of noise at a train manufacturer was a study that considered the way to measure the noise, in which workers would be evaluated, as well as other criteria.
\end{abstract}

KEYWORDS: Noise, occupational; Hearing loss, noise-induced; Occupational exposure.

\title{
REFERENCIAS
}

1. Brevigliero E, Possebon J, Spinelli R. Higiene ocupacional, agentes biológicos, químicos e físicos. 4a ed. São Paulo: SENAC; 2010

2. Guida HL, Morini RG, Cardoso ACV. Avaliação audiológica em trabalhadores expostos a ruído e praguicida. Braz J Otorhinolaryngol. 2010;76(4):423-7. DOI: http://dx.doi. org/10.1590/S1808-86942010000400003

3. Fernandes JC. Avaliação da perda de audição induzida por ruído em trabalhadores expostos a níveis inferiores 85 dB(a). In: Anais do Simpósio de Engenharia de Produção; 08-10 nov. 1999; Bauru, Brasil. Bauru: UNESP; 1999.

4. Macedo ISC, Mateus DC, Costa EDMGCC, Asprino ACL, Lourenço EA. Avaliação do ruído em unidades de terapia intensiva. Braz J Otorhinolaryngol. 2009;75(6):844-6. DOI: http://dx.doi.org/10.1590/S1808-86942009000600012

5. Cordeiro R, Clemente APG, Diniz CS, Dias A. Exposição ao ruído ocupacional como fator de risco para acidentes de trabalho. Rev Saúde Pública. 2005;39(3):461-6. DOI: http://dx.doi.org/10.1590/S0034-89102005000300018

6. De Faria CAR, Suzuki FA. Avaliação dos limiares auditivos com e sem equipamento de proteção individual. Rev Bras Otorrinolaringol. 2008;74(3):417-22. DOI: http://dx.doi. org/10.1590/S0034-72992008000300017

7. Harger MRHC, Branco AB. Efeitos auditivos decorrentes da exposição ocupacional ao ruído em trabalhadores de marmorarias no Distrito Federal. Rev Assoc Med Bras. 2004;50(4):396-9. DOI: http://dx.doi.org/10.1590/S010442302004000400029

8. Brasil. Norma Regulamentadora 15 - Atividades e operações insalubres. Portaria MTb n. ${ }^{\circ} 3.214$, de 08 de junho de 1978. Disponível em: http://portal.mte.gov.br/ data/files/FF8080812DF396CA012E0017BB3208E8/ NR-15\%20(atualizada 2011).pdf

9) Pinto ALT, Windt MCVS, Céspedes L. Segurança em medicina do trabalho. 5a ed. São Paulo: Saraiva; 2010.

10. Brasil. Ministério do Trabalho e Emprego. Portaria $n^{\circ} 19$, de 9 de abril de 1998. Estabelece diretrizes e parâmetros mínimos para avaliação e acompanhamento da audição em trabalhadores expostos a níveis de pressão sonora elevados. Disponível em: http://portal.mte.gov.br/data/files/FF80808 12BE914E6012BEEB7F30751E6/p_19980409_19.pdf.

11. Brasil. Ministério da Previdência Social. Disponível em: http:/www.previdencia.gov.br.

12. Brasil. Associação Nacional de Medicina do Trabalho. Disponível em: http://www.anamt.org.br/

13. Brasil. Associação Nacional de Medicina do Trabalho. Disponível em: http://portalsaude.saude.gov.br/portalsaude/ index.html 